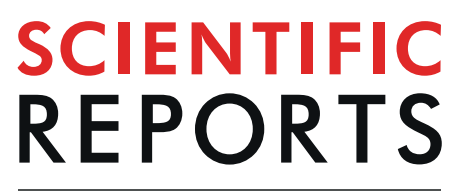

natureresearch

\title{
OPEN First-principles investigation on electronic properties and band alignment of group III monochalcogenides
}

Received: 21 April 2019

Accepted: 15 August 2019

Published online: 16 September 2019

\author{
Chongdan Ren ${ }^{1}$, Sake Wang ${ }^{2}$, Hongyu Tian ${ }^{3}$, Yi Luo $\mathbb{1}^{4}$, Jin Yu ${ }^{4}$, Yujing Xu ${ }^{5}$ \& Minglei Sun $\mathbb{1}^{5}$ \\ Using first-principles calculations, we investigated the electronic properties and band alignment of \\ monolayered group III monochalcogenides. First, we calculated the structural and electronic properties \\ of six group III monochalcogenides ( $\mathrm{GaS}, \mathrm{GaSe}, \mathrm{GaTe}, \mathrm{InS}$, InSe, and InTe). We then investigated \\ their band alignment and analysed the possibilities of forming type-I and type-II heterostructures by \\ combining these compounds with recently developed two-dimensional (2D) semiconducting materials, \\ as well as forming Schottky contacts by combining the compounds with 2D Dirac materials. We aim \\ to provide solid theoretical support for the future application of group III monochalcogenides in \\ nanoelectronics, photocatalysis, and photovoltaics.
}

Group III monochalcogenides $\left(\mathrm{M}_{\mathrm{III}} \mathrm{Xs}\right.$, where $\mathrm{M}_{\mathrm{III}}$ represents a group III element and X represents a chalcogen), a family of monolayered semiconducting materials, have attracted much research interest in recent years ${ }^{1-8}$. $\mathrm{M}_{\mathrm{III}} \mathrm{Xs}$ are semiconductors with moderate bandgaps which are sensitive to the number of layers in the material ${ }^{9}$. Their suitability for use as transistors ${ }^{10}$, sensors ${ }^{11}$, and photodetectors ${ }^{12-16}$ has been addressed in a number of studies. For example, Lei et al. ${ }^{13}$ fabricated an ultrathin InSe-based photodetector whose overall performance surpassed those of similar devices. Many researchers also investigated the effects of doping ${ }^{17}$, defects ${ }^{18}$, applied elastic strain $^{19}$, and an external electric field ${ }^{20}$ on the electronic and optical properties of $\mathrm{M}_{\mathrm{III}} \mathrm{X}$ s. More importantly, many types of $\mathrm{M}_{\mathrm{III}} \mathrm{X}$ have been synthesized ${ }^{21}$. All these investigations demonstrate that $\mathrm{M}_{\mathrm{III}} \mathrm{X}$ s can be an important category of 2D semiconductor materials for application in many fields.

In this paper, we report the results of our comprehensive investigation on the electronic properties and band alignment of $\mathrm{M}_{\mathrm{III}} \mathrm{Xs}\left(\mathrm{M}_{\mathrm{III}}=\mathrm{Ga}\right.$ or In, $\mathrm{X}=\mathrm{S}$, Se, or Te; including GaS, GaSe, GaTe, InS, InSe, and InTe). More specifically, the structural parameters, band structures, and band edges were calculated for each material. We then explored the possibility of these materials forming type I, II, and III heterostructures with popular 2D semiconducting materials, including $\mathrm{MoS}_{2}, \mathrm{MoSe}_{2}, \mathrm{WS}_{2}$, WSe 2 , black phosphorene, blue phosphorene, arsenene, h-BN, $\mathrm{g}-\mathrm{GaN}$, and germanane; the results are reported here. In addition, we also report our analysis of the possibility to form Schottky contacts between $\mathrm{M}_{\mathrm{III}} \mathrm{Xs}$ and 2D Dirac materials such as graphene and silicene. Our results will not only provide basic information on the properties of $\mathrm{M}_{\mathrm{III}} \mathrm{Xs}$, but also fundamental guidelines for future application of $\mathrm{M}_{\mathrm{III}} \mathrm{Xs}$.

\section{Calculation Details}

We used density-functional theory with the Perdew-Burke-Ernzerhof functional ${ }^{22}$ and projector-augmented waves $^{23,24}$ to treat the valence electrons as implemented in the Vienna Ab Initio Simulation Package (VASP) ${ }^{25,26}$. The hybrid Heyd-Scuseria-Ernzerhof (HSE06) functional ${ }^{25}$ was also selected to compute the electronic properties. The mixing parameter was set to 0.25 , while the screening parameter was set to $0.2 \AA^{-1}$. The zero-damping $\mathrm{vdW}-\mathrm{D} 3$ correction proposed by Grimme ${ }^{26}$ was used to describe the long-range interaction. The energy cutoff for

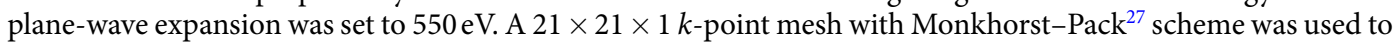

${ }^{1}$ Department of Physics, Zunyi Normal College, Zunyi, Guizhou, 563002, China. ${ }^{2}$ College of Science, Jinling Institute of Technology, Nanjing, Jiangsu, 211169, China. ${ }^{3}$ School of Physics and Electronic Engineering, Linyi University, Linyi, Shandong, 276005, China. ${ }^{4}$ School of Materials Science and Engineering, Southeast University, Nanjing, Jiangsu, 211189, China. ${ }^{5}$ School of Mechanical Engineering, Southeast University, Nanjing, Jiangsu, 211189, China. Correspondence and requests for materials should be addressed to C.R. (email: renchongdan@hotmail.com) or M.S. (email: mignleisun@outlook.com) 


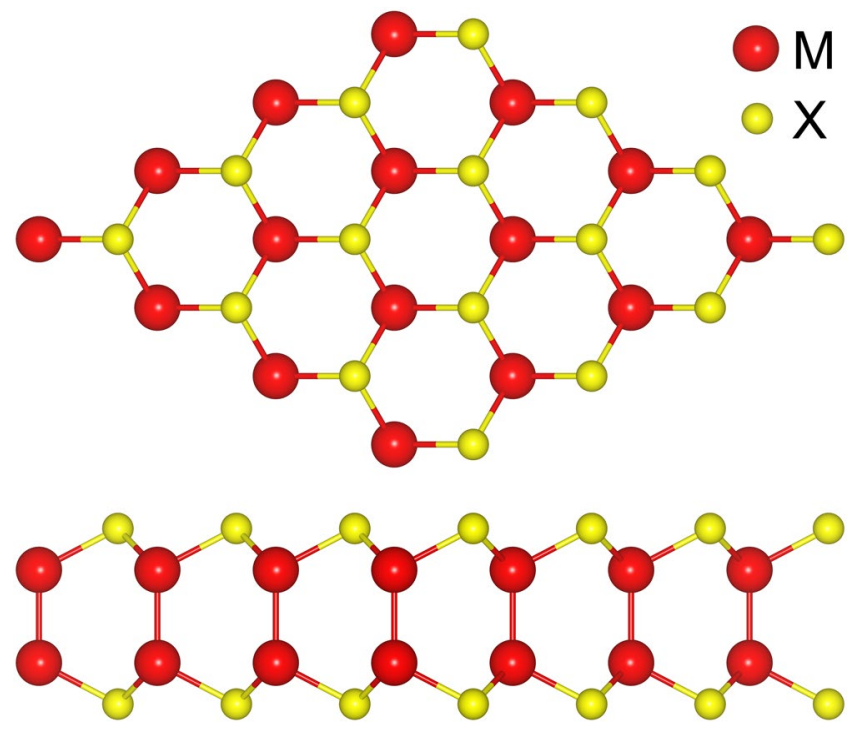

Figure 1. Crystal structure of $\mathrm{M}_{\mathrm{III}} \mathrm{Xs}$ examined in this study: GaS, GaSe, GaTe, InS, InSe, and InTe.

sample the first Brillouin zone. The tetrahedron methodology with Blöchl corrections ${ }^{28}$ was used for all the calculations, only except the Gaussian smearing methodology ${ }^{29}$ with a smearing of $0.01 \mathrm{eV}$ was employed for band structure calculations. To avoid interaction between adjacent images, a relatively large vacuum space of $20 \AA$ was inserted in the normal direction. After the fully relaxation, the force on each atom was less than $0.01 \mathrm{~V} / \AA$. All the calculations were performed in a spin-restricted manner.

\section{Results and Discussions}

The crystal structure of $\mathrm{M}_{\mathrm{III}} \mathrm{X}$ is shown in Fig. 1. A $\mathrm{M}_{\mathrm{III}} \mathrm{X}$ monolayer is formed by four covalently bonded atomic planes in an X-M $-\mathrm{M}_{\mathrm{III}}-\mathrm{M}_{\mathrm{III}}-\mathrm{X}$ sequence. The relaxed lattice constants of $\mathrm{GaS}, \mathrm{GaSe}, \mathrm{GaTe}, \mathrm{InS}$, InSe, and InTe are $3.62,3.80,4.12,3.91,4.06$, and $4.36 \AA$, respectively. Meanwhile, the thicknesses of GaS, GaSe, GaTe, InS, InSe, and InTe are $4.65,4.80,4.99,5.19,5.37$, and $5.56 \AA$, respectively.

The band structures of the $\mathrm{M}_{\mathrm{II}} \mathrm{Xs}$ are shown in Fig. 2. All of the $\mathrm{M}_{\mathrm{II}} \mathrm{X}$ monolayers are indirect-bandgap semiconductors. For each $\mathrm{M}_{\mathrm{III}} \mathrm{X}$ monolayer, the valence-band maximum (VBM) is located between the $\Gamma$ and $\mathrm{M}$ points; however, the positions of their conduction-band minimum (CBM) are slightly different. For GaS and $\mathrm{GaTe}$, their CBM are located at their respective M points. Meanwhile, for $\mathrm{GaSe}$, InS, InSe, and InTe, their CBM are located at their respective $\Gamma$ points. Using the HSE06 functional, the calculated gap values for GaS, GaSe, GaTe, InS, InSe, and InTe monolayer are 3.29, 2.77, 2.13, 2.63, 2.30, and $2.07 \mathrm{eV}$, respectively.

We observed an interesting feature in the band structures of these $\mathrm{M}_{\mathrm{III}} \mathrm{Xs}$ - band convergence. For all of the $\mathrm{M}_{\mathrm{III}} \mathrm{X}$ monolayers, in addition to the VBM located at the $\Gamma-\mathrm{M}$ high-symmetry line, there is another valley in the valence band along the $\mathrm{K}-\Gamma$ high-symmetry line. The difference between the energies of these two valleys is only $4,25,34,18,18$, and $3 \mathrm{meV}$ for the GaS, GaSe, GaTe, InS, InSe, and InTe monolayers, respectively, which are all much lower than $52 \mathrm{meV}\left(2 k_{\mathrm{B}} T_{300 \mathrm{~K}}\right.$, i.e. twice the thermal energy at room temperature). This means that band convergence may occur in the valence band of $\mathrm{GaS}, \mathrm{GaSe}, \mathrm{GaTe}, \mathrm{InS}, \mathrm{InSe}$, and InTe. When both valleys contribute to the total electrical conductivity $(\sigma)$, the power factor $\left(P=S^{2} \sigma\right.$, where $S$ presents the Seebeck coefficient) is considerably increased. Since the Seebeck coefficient is one of the main factors of a material's ability to efficiently produce thermoelectric power, the band convergence in the GaS, GaSe, GaTe, InS, InSe, and InTe monolayers may allow them to be used as thermoelectric materials, as previously shown for $\mathrm{MoS}_{2}{ }^{30-32}$ and phosphorene ${ }^{33}$. Indeed, Tung et al. ${ }^{34}$ found that the maximum $P$ of a p-type (and n-type) InSe monolayer can reach 0.049 (and $0.043) \mathrm{W} / \mathrm{K}^{2} \mathrm{~m}$ at $300 \mathrm{~K}$ in the armchair direction.

The band alignment of $\mathrm{M}_{\mathrm{III}} \mathrm{Xs}$ is shown in Fig. 3. The energy levels of CBM and VBM were calculated with reference to the

vacuum level, while the vacuum level was determined through the calculation of the planar averaged electrostatic potential. The VBM values of GaS, GaSe, GaTe, InS, InSe, and InTe monolayers are -6.88, -6.40, -5.78, $-7.02,-6.56$, and $-5.91 \mathrm{eV}$, respectively. Meanwhile, the CBM values of GaS, GaSe, GaTe, InS, InSe, and InTe monolayers are $-3.59,-3.63,-3.65,-4.38,-4.26$, and $-3.84 \mathrm{eV}$, respectively. As reported in a previous investigation $^{35}$, the reduction potential $\left(\mathrm{E}_{\mathrm{H}^{+} / \mathrm{H}_{2}}\right)$ and oxidation potential $\left(\mathrm{E}_{\mathrm{O}_{2} / \mathrm{H}_{2} \mathrm{O}}\right)$ of water are -4.44 and $-5.67 \mathrm{eV}$, respectively. These values lie just within the bandgaps of the GaS, GaSe, GaTe, InS, InSe, and InTe monolayers, suggesting that these $2 \mathrm{D}$ materials can potentially serve as photocatalysts for water splitting, as previously reported by Zhuang et al. ${ }^{2}$.

Recent studies show that 2D-material-based Schottky contacts have great potential in nanoelectronic devices $^{36-38}$ and sensors ${ }^{39}$. To explore the opportunities of forming a Schottky contact between each $\mathrm{M}_{\mathrm{II}} \mathrm{X}$ monolayer and each 2D Dirac material, we calculated and obtained values of 4.23 and $4.60 \mathrm{eV}$ as the the work functions of graphene and silicene, respectively. Figure 3 shows that graphene can form n-type Schottky contacts 

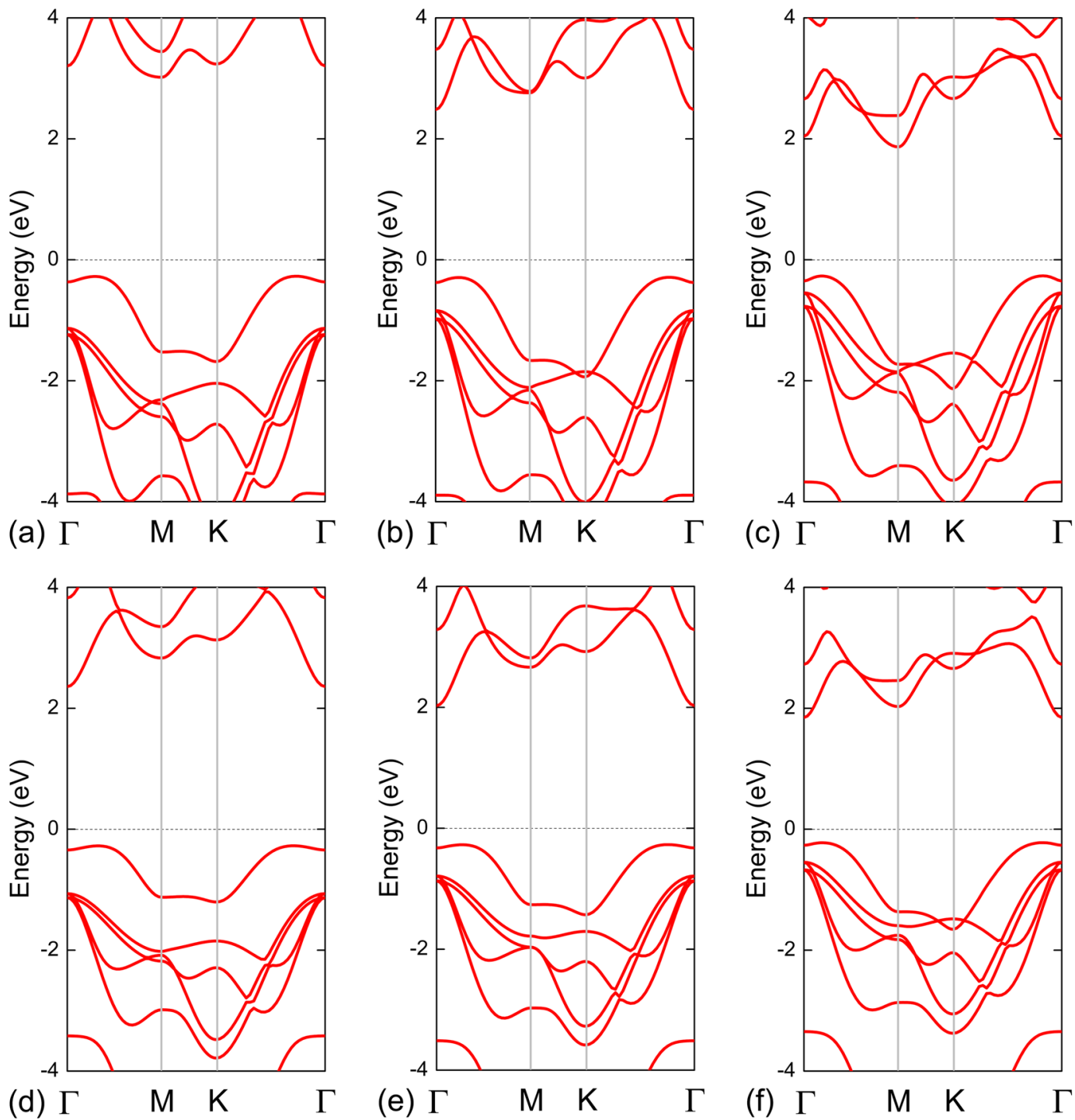

Figure 2. Band structures of (a) GaS, (b) GaSe, (c) GaTe, (d) InS, (e) InSe, and (f) InTe monolayers, obtained using the HSE06 functional. The black dashed line denotes the Fermi level.

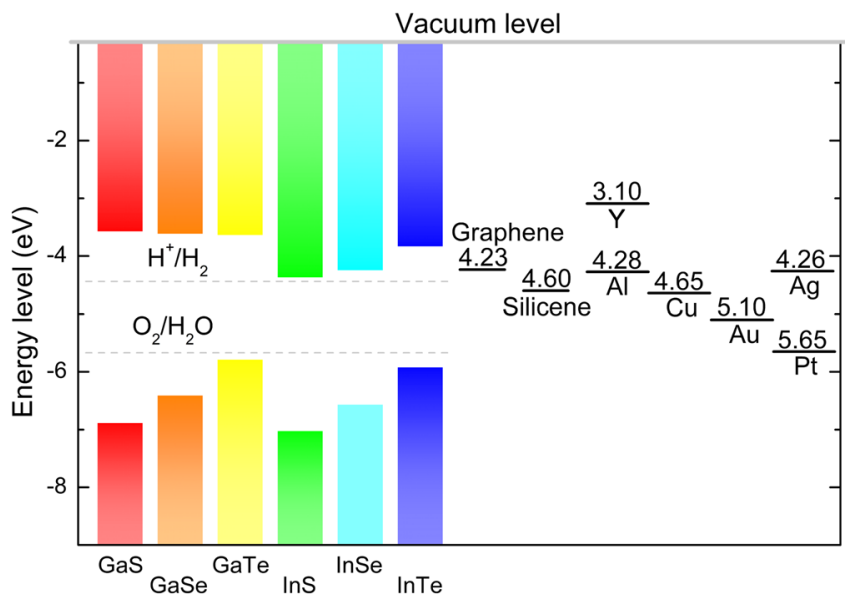

Figure 3. Band alignment of GaS, GaSe, GaTe, InS, InSe, and InTe monolayers. The energy of the vacuum level was set to zero. The work functions of graphene and silicene, as well as those of elemental Y, $\mathrm{Al}, \mathrm{Cu}, \mathrm{Ag}, \mathrm{Au}$, and $\mathrm{Pt}$ are also shown; the work functions of $\mathrm{Y}, \mathrm{Al}, \mathrm{Cu}, \mathrm{Ag}, \mathrm{Au}$, and $\mathrm{Pt}$ were obtained from experimental data $\mathrm{in}^{43}$. 


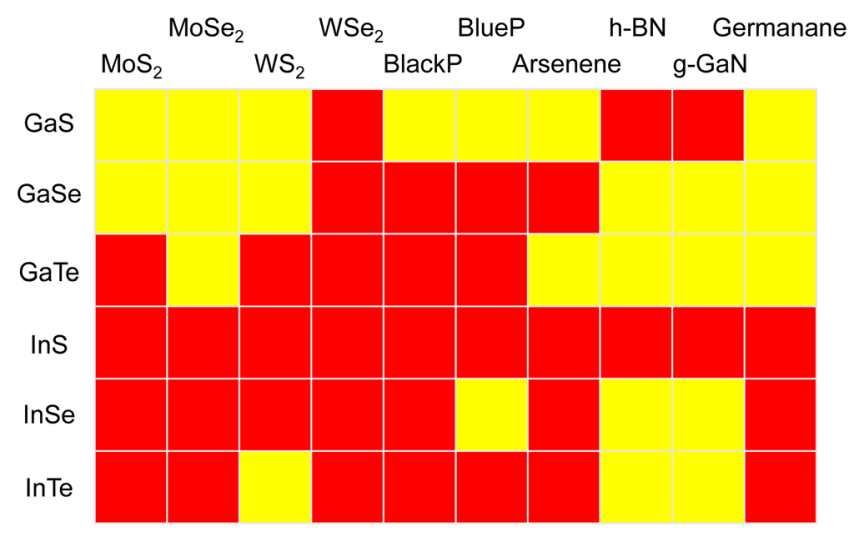

Figure 4. Table of heterostructures formed between $\mathrm{M}_{\mathrm{III}} \mathrm{Xs}$ (GaS, GaSe, GaTe, InS, InSe, and InTe) and various $2 \mathrm{D}$ semiconducting materials $\left(\mathrm{MoS}_{2}, \mathrm{MoSe}_{2}, \mathrm{WS}_{2}, \mathrm{WSe}\right.$, BlackP, BlueP, arsenene, h-BN, g-GaN, and germanane). Type-I and type-II heterostructures are shown in yellow and red, respectively.

with GaS, GaSe, GaTe, and InTe, and n-type Ohmic contacts with InS and InSe. These predictions are in good agreement with results of recent studies ${ }^{40,41}$. Meanwhile, silicene can form n-type Schottky contacts with GaS, GaSe, GaTe, InS, InSe, and InTe. Overall, our findings are expected to be useful to the design of Schottky devices with dedicated Schottky barrier height.

In addition, $\mathrm{p}-\mathrm{n}$ junctions are fimportant for building nanoelectronic devices. Control of the carrier type in $2 \mathrm{D}$ semiconducting materials is a fundamental requirement for the application of nanoelectronic devices in various fields. Peng et al. ${ }^{42}$ developed a method for direct inject the carrier into a nanochannel by using a metal electrode with proper work function. That is a p-type (or n-type) device channel can be obtained by chosing a metal electrode with higher (or lower) work function than that in the channel. Figure 3 shows the band alignment of GaS, GaSe, GaTe, InS, InSe, and InTe. The work functions of widely used metals for electrode like Y, Al, Cu, $\mathrm{Ag}, \mathrm{Au}$, and $\mathrm{Pt}$ are also shown for comparison ${ }^{43}$. Obviously, when $\mathrm{Al}$ and $\mathrm{Ag}$ are used as the electrode material, both the $\mathrm{Al} / \mathrm{InS}$ and $\mathrm{Ag} / \mathrm{InS}$ interfaces may form an Ohmic contact. Furthermore, when $\mathrm{Y}$ is used as the electrode material, all of the $\mathrm{Y} / \mathrm{M}_{\mathrm{III}} \mathrm{X}$ interfaces are highly probably form an Ohmic contacts, inducing effective carrier injection as well as enhancement of contact performance.

The main obstacle to the application of a material in photocatalysis is the short life time of photo-generated carriers. Generally, forming a van der Waals (vdW) heterostructure with type-II band alignment can significantly extend the life time of photo-generated carriers ${ }^{44-51}$. We systematically investigated the possibility of forming type-II heterostructures by combining $\mathrm{M}_{\mathrm{III}} \mathrm{Xs}$ with other popular $2 \mathrm{D}$ semiconducting materials, including $\mathrm{MoS}_{2}$, $\mathrm{MoSe}_{2}, \mathrm{WS}_{2}, \mathrm{WSe}_{2}$, black phosphorene (BlackP), blue phosphorene (BlueP), arsenene, h-BN, g-GaN, and germanane; the results are presented in Fig. 4 . In brief, $\mathrm{GaS}$ can form type-II heterostructures with WSe $\mathrm{H}_{2} \mathrm{~h}-\mathrm{BN}$, and g-GaN; GaSe can form type-II heterostructures with WSe ${ }_{2}$, BlackP, BlueP, and arsenene; GaTe can form type-II heterostructures with $\mathrm{MoS}_{2}, \mathrm{WS}_{2}, \mathrm{WSe}_{2}$, BlackP, and BlueP; InS can form type-II heterostructures with all of the $2 \mathrm{D}$ semiconducting materials examined in our study; InSe can form type-II heterostructures with $\mathrm{MoS}_{2}$, $\mathrm{MoSe}_{2}, \mathrm{WS}_{2}, \mathrm{WSe}_{2}$, BlackP, arsenene, and germanane; and InTe can form type-II heterostructures with $\mathrm{MoS}_{2}$, $\mathrm{MoSe}_{2}$, WSe $\mathrm{W}_{2}$, BlackP, BlueP, arsenene, and germanane. These results can provide useful guidelines for designing high-efficiency $\mathrm{M}_{\mathrm{III}} \mathrm{X}$-based photocatalysts for water splitting.

Using first-principles calculations, we systematically investigated the electronic properties and band alignment of a family of 2D semiconducting materials-group III monochalcogenides (GaS, GaSe, GaTe, InS, InSe, and InTe). We found that all six $\mathrm{M}_{\mathrm{III}} \mathrm{X}$ materials are indirect-bandgap semiconducting materials (the bandgaps of GaS, GaSe, GaTe, InS, InSe, and InTe are 3.29, 2.77, 2.13, 2.63, 2.30, and $2.07 \mathrm{eV}$, respectively). Interestingly, we discovered band convergence in all of the $\mathrm{M}_{\mathrm{III}} \mathrm{X}$ materials, indicating their potential for thermoelectric applications. The calculated results for band alignment of the $\mathrm{M}_{\mathrm{III}} \mathrm{Xs}$ indicate that all of the $\mathrm{M}_{\mathrm{III}} \mathrm{X}$ monolayers are potential photocatalysts for water splitting. Moreover, the $\mathrm{M}_{\mathrm{III}} \mathrm{X}$ monolayers can form type-II heterostructures with other popular $2 \mathrm{D}$ semiconducting materials, which is a critical requirement for photocatalyst and photovoltaic applications. We also found that most $\mathrm{M}_{\mathrm{III}} \mathrm{X}$ monolayers can form n-type Schottky contacts with graphene and silicene. In addition, when elemental $\mathrm{Y}$ is used as the electrode material, all of the $\mathrm{Y} / \mathrm{M}_{\mathrm{III}} \mathrm{X}$ interfaces may form Ohmic contacts. We believe our findings can help to extend the application of group III monochalcogenides in thermoelectrics, photocatalysis, photovoltaics, and nanoelectronics.

\section{References}

1. Demirci, S., Avazli, N., Durgun, E. \& Cahangirov, S. Structural and electronic properties of monolayer group III monochalcogenides. Phys. Rev. B 95, 115409 (2017).

2. Zhuang, H. L. \& Hennig, R. G. Single-Layer Group-III Monochalcogenide Photocatalysts for Water Splitting. Chem. Mater. 25, 3232-3238 (2013).

3. Cai, Y., Zhang, G. \& Zhang, Y.-W. Charge Transfer and Functionalization of Monolayer InSe by Physisorption of Small Molecules for Gas Sensing. J. Phys. Chem. C 121, 10182-10193 (2017).

4. Zhou, H., Cai, Y., Zhang, G. \& Zhang, Y.-W. Unusual phonon behavior and ultra-low thermal conductance of monolayer InSe. Nanoscale 10, 480-487 (2018). 
5. Zhu, Z., Cheng, Y. \& Schwingenschlögl, U. Topological Phase Transition in Layered GaS and GaSe. Phys. Rev. Lett. 108, 266805 (2012).

6. Zhang, Q. \& Schwingenschlögl, U. Rashba effect and enriched spin-valley coupling in Ga $X / M X(M=\mathrm{Mo}, \mathrm{W} ; X=\mathrm{S}, \mathrm{Se}, \mathrm{Te})$ heterostructures. Phys. Rev. B 97, 155415 (2018).

7. Pham, K. D. et al. Ab-initio study of electronic and optical properties of biaxially deformed single-layer GeS. Superlattices Microstruct. 120, 501-507 (2018).

8. Khoa, D. Q. et al. Modulation of electronic properties of monolayer InSe through strain and external electric field. Chem. Phys. 516, 213-217 (2019)

9. Li, Y., Chen, H., Huang, L. \& Li, J. Ab Initio Study of the Dielectric and Electronic Properties of Multilayer GaS Films. J. Phys. Chem. Lett. 6, 1059-1064 (2015).

10. Kuc, A. et al. High-Performance 2D p-Type Transistors Based on GaSe Layers: An Ab Initio Study. Adv. Electron. Mater. 3, 1600399 (2017).

11. Li, W. \& Li, J. Piezoelectricity in two-dimensional group-III monochalcogenides. Nano Res. 8, 3796-3802 (2015).

12. Lei, S. et al. Evolution of the Electronic Band Structure and Efficient Photo-Detection in Atomic Layers of InSe. ACS Nano 8 , 1263-1272 (2014).

13. Lei, S. et al. An Atomically Layered InSe Avalanche Photodetector. Nano Lett. 15, 3048-3055 (2015).

14. Luo, W. et al. Gate Tuning of High-Performance InSe-Based Photodetectors Using Graphene Electrodes. Adv. Opt. Mater. 3, 1418-1423 (2015).

15. Huang, H. et al. Highly sensitive phototransistor based on GaSe nanosheets. Appl. Phys. Lett. 107, 143112 (2015).

16. Cao, Y. et al. Strong enhancement of photoresponsivity with shrinking the electrodes spacing in few layer GaSe photodetectors. Sci. Rep. 5, 8130 (2015).

17. Chen, H., Li, Y., Huang, L. \& Li, J. Influential Electronic and Magnetic Properties of the Gallium Sulfide Monolayer by Substitutional Doping. J. Phys. Chem. C 119, 29148-29156 (2015).

18. Guo, Y., Zhou, S., Bai, Y. \& Zhao, J. Defects and oxidation of group-III monochalcogenide monolayers. J. Chem. Phys. 147, 104709 (2017).

19. Huang, L., Chen, Z. \& Li, J. Effects of strain on the band gap and effective mass in two-dimensional monolayer $\mathrm{Ga} X(X=\mathrm{S}, \mathrm{Se}, \mathrm{Te})$. RSC Adv. 5, 5788-5794 (2015).

20. Guo, F. et al. Modulation of Electronic and Optical Anisotropy Properties of ML-GaS by Vertical Electric Field. Nanoscale Res. Lett. 12, 409 (2017).

21. Xu, K. et al. Synthesis, properties and applications of 2D layered M X ( $M=G a, I n ; X=S, S e, T e)$ materials. Nanoscale 8, 16802-16818 (2016).

22. Perdew, J. P., Burke, K. \& Ernzerhof, M. Generalized Gradient Approximation Made Simple. Phys. Rev. Lett. 77, 3865-3868 (1996).

23. Blöchl, P. E. Projector augmented-wave method. Phys. Rev. B 50, 17953-17979 (1994).

24. Kresse, G. \& Joubert, D. From ultrasoft pseudopotentials to the projector augmented-wave method. Phys. Rev. B 59, 1758-1775 (1999).

25. Kresse, G. \& Furthmüller, J. Efficiency of ab-initio total energy calculations for metals and semiconductors using a plane-wave basis set. Comput. Mater. Sci. 6, 15-50 (1996).

26. Kresse, G. \& Furthmüller, J. Efficient iterative schemes for ab initio total-energy calculations using a plane-wave basis set. Phys. Rev. B 54, 11169-11186 (1996).

27. Monkhorst, H. J. \& Pack, J. D. Special points for Brillouin-zone integrations. Phys. Rev. B 13, 5188 (1976)

28. Blöchl, P. E., Jepsen, O. \& Andersen, O. K. Improved tetrahedron method for Brillouin-zone integrations. Phys. Rev. B 49, 16223-16233 (1994).

29. Fu, C. L. \& Ho, K. M. First-principles calculation of the equilibrium ground-state properties of transition metals: Applications to Nb and Mo. Phys. Rev. B 28, 5480-5486 (1983).

30. Hong, J., Lee, C., Park, J.-S. \& Shim, J. H. Control of valley degeneracy in $\mathrm{MoS}_{2}$ by layer thickness and electric field and its effect on thermoelectric properties. Phys. Rev. B 93, 035445 (2016)

31. Hippalgaonkar, K. et al. High thermoelectric power factor in two-dimensional crystals of $\mathrm{MoS}_{2}$. Phys. Rev. B 95, 115407 (2017).

32. Zhang, G. \& Zhang, Y.-W. Thermoelectric properties of two-dimensional transition metal dichalcogenides. J. Mater. Chem. C 5, 7684-7698 (2017).

33. Lv, H. Y., Lu, W. J., Shao, D. F. \& Sun, Y. P. Enhanced thermoelectric performance of phosphorene by strain-induced band convergence. Phys. Rev. B 90, 085433 (2014).

34. Hung, N. T., Nugraha, A. R. \& Saito, R. Two-dimensional InSe as a potential thermoelectric material. Appl. Phys. Lett. 111, 092107 (2017).

35. Weast, R. C. Handbook of Physics and Chemistry. 1983-1984 (CRC Press, 1986).

36. Yang, H. et al. Graphene Barristor, a Triode Device with a Gate-Controlled Schottky Barrier. Science 336, 1140-1143 (2012).

37. Nie, Y., Hong, S., Wallace, R. M. \& Cho, K. Theoretical Demonstration of the Ionic Barristor. Nano Lett. 16, 2090-2095 (2016).

38. Shih, C.-J. et al. Tuning On-Off Current Ratio and Field-Effect Mobility in a $\mathrm{MoS}_{2}-$ Graphene Heterostructure via Schottky Barrier Modulation. ACS Nano 8, 5790-5798 (2014).

39. Liu, B. et al. High-Performance Chemical Sensing Using Schottky-Contacted Chemical Vapor Deposition Grown Monolayer $\mathrm{MoS}_{2}$ Transistors. ACS Nano 8, 5304-5314 (2014).

40. Phuc, H., Nguyen, H., Hoi, B. D. \& Nguyen, C. Interlayer coupling and electric field tunable electronic properties and Schottky barrier in graphene/bilayer-GaSe van der Waals heterostructure. Phys. Chem. Chem. Phys. 20, 17899-17908 (2018).

41. Pham, K. D. et al. First principles study on the electronic properties and Schottky barrier of Graphene/InSe heterostructure. Superlattices Microstruct. 122, 570-576 (2018).

42. Peng, L. M., Zhang, Z., Wang, S. \& Liang, X. A doping-free approach to carbon nanotube electronics and optoelectronics. AIP Adv. 2, 041403 (2012).

43. Michaelson, H. B. The work function of the elements and its periodicity. J. Appl. Phys. 48, 4729-4733 (1977).

44. Özçelik, V. O., Azadani, J. G., Yang, C., Koester, S. J. \& Low, T. Band alignment of two-dimensional semiconductors for designing heterostructures with momentum space matching. Phys. Rev. B 94, 035125 (2016).

45. Sun, M., Chou, J.-P., Yu, J. \& Tang, W. Electronic properties of blue phosphorene/graphene and blue phosphorene/graphene-like gallium nitride heterostructures. Phys. Chem. Chem. Phys. 19, 17324-17330 (2017).

46. Wang, S., Ren, C., Tian, H., Yu, J. \& Sun, M. MoS2/ZnO van der Waals heterostructure as a high-efficiency water splitting photocatalyst: a first-principles study. Phys. Chem. Chem. Phys. 20, 13394-13399 (2018).

47. Din, H. U. et al. Electronic structure, optical and photocatalytic performance of $\mathrm{SiC}-\mathrm{MX} 2(\mathrm{M}=\mathrm{Mo}, \mathrm{W}$ and $\mathrm{X}=\mathrm{S}, \mathrm{Se})$ van der Waals heterostructures. Phys. Chem. Chem. Phys. 20, 24168-24175 (2018).

48. Wang, B.-J. et al. Electronic structures and enhanced photocatalytic properties of blue phosphorene/BSe van der Waals heterostructures. J. Mater. Chem. A 6, 8923-8929 (2018).

49. Wang, S., Tian, H., Ren, C., Yu, J. \& Sun, M. Electronic and optical properties of heterostructures based on transition metal dichalcogenides and graphene-like zinc oxide. Sci. Rep. 8, 12009 (2018).

50. Li, S. et al. First-principles calculations of the electronic properties of SiC-based bilayer and trilayer heterostructures. Phys. Chem. Chem. Phys. 20, 24726-24734 (2018).

51. You, B., Wang, X., Zheng, Z. \& Mi, W. Black phosphorene/monolayer transition-metal dichalcogenides as two dimensional van der Waals heterostructures: a first-principles study. Phys. Chem. Chem. Phys. 18, 7381-7388 (2016). 


\section{Acknowledgements}

This study was funded by the National Science Foundation for Young Scientists of China (grant number 11704165), the National Natural Science Foundation of China (grant number 11864047), the Major Research Project for Innovative Group of Education Department of Guizhou Province (grant number KY[2018]028), the Science Foundation of Guizhou Provincial Education Department (grant number QJHKYZ[2016]092), as well as the Science Foundation of Jinling Institute of Technology (grant number 40620064). The computational resources utilized in this research were provided by the National Supercomputing Center in Wuxi, China.

\section{Author Contributions}

Minglei Sun supervised the project. Chongdan Ren and Minglei Sun wrote the main manuscript text. Chongdan Ren, Sake Wang, Hongyu Tian, Yi Luo, Jin Yu, Yujing Xu, and Minglei Sun read and approved the final manuscript.

\section{Additional Information}

Competing Interests: The authors declare no competing interests.

Publisher's note Springer Nature remains neutral with regard to jurisdictional claims in published maps and institutional affiliations.

(c) (i) Open Access This article is licensed under a Creative Commons Attribution 4.0 International License, which permits use, sharing, adaptation, distribution and reproduction in any medium or format, as long as you give appropriate credit to the original author(s) and the source, provide a link to the Creative Commons license, and indicate if changes were made. The images or other third party material in this article are included in the article's Creative Commons license, unless indicated otherwise in a credit line to the material. If material is not included in the article's Creative Commons license and your intended use is not permitted by statutory regulation or exceeds the permitted use, you will need to obtain permission directly from the copyright holder. To view a copy of this license, visit http://creativecommons.org/licenses/by/4.0/.

(c) The Author(s) 2019 